Check for updates

Cite this: RSC Adv., 2018, 8, 17025

Received 28th February 2018

Accepted 27th April 2018

DOI: 10.1039/c8ra01761a

rsc.li/rsc-advances

\section{$\delta$-Carboline-based bipolar host materials for deep blue thermally activated delayed fluorescence OLEDs with high efficiency and low roll-off characteristic $\dagger$}

\author{
Ji Su Moon, $\$ \S$ Dae Hyun Ahn, $\$$ Si Woo Kim, $\$$ S Seung Yeon Lee, $\$ \S$ Ju Young Lee $\$ \S^{\star}$ \\ and Jang Hyuk Kwon (D) $\$ \delta^{*}$
}

Two bipolar host materials, 8-(9H-carbazol-9-yl)-5-(pyridin-2-yl)-5H-pyrido[3,2-b]indole (CzCbPy) and 5(6-(9H-carbazol-9-yl)pyridin-2-yl)-8-(9H-carbazol-9-yl)-5H-pyrido[3,2-b]indole (2CzCbPy), were synthesized for deep blue thermally activated delayed fluorescence organic light emitting diodes (TADF OLEDs). Both $\mathrm{CzCbPy}$ and $2 \mathrm{Cz} C b P y$ hosts possess bipolar characteristic with high polarity, which results in high delayed photoluminescence quantum yields by reducing the energy gap between singlet and triplet states of TADF materials. In addition, these hosts have high enough triplet energies of $3.05 \mathrm{eV}$ to transfer exciton energy to a deep blue TADF emitter. A deep blue TADF OLED fabricated with a $\mathrm{CzCbPy}$ host exhibited high external quantum efficiency of $22.9 \%$ and low efficiency roll-off $\left(19.2 \%\right.$ at $\left.1000 \mathrm{~cd} \mathrm{~m}^{-2}\right)$.

\section{Introduction}

Thermally activated delayed fluorescence (TADF) OLEDs have recently attracted great attention because they can achieve a theoretical maximum internal quantum efficiency (IQE) of $100 \%$ by harvesting both singlet and triplet excitons for light emission. ${ }^{1-6}$ In TADF OLEDs, triplet excitons are readily upconverted into the singlet state by reverse intersystem crossing (RISC) owing to extremely small singlet-triplet energy differences, then all singlet excitons can emit either prompt fluorescence or delayed fluorescence. ${ }^{7,8}$ To date, a lot of TADF materials with external quantum efficiencies (EQEs) of over $20 \%$ have been reported. ${ }^{9-11}$ However, many TADF OLEDs, specially blue TADF OLEDs, have a problem of high efficiency roll-off due to the exciton-polaron quenching and triplet-triplet annihilation (TTA). To avoid this problem and achieve high efficiency, TADF materials are usually dispersed in a suitable host matrix at a relatively low concentration. Hence, an ideal host should possess high triplet energy $\left(T_{1}\right)$, appropriate frontier molecular

Department of Information Display, Kyung Hee University, 26 Kyungheedae-ro, Dongdaemoon-gu, Seoul, 130-701, Republic of Korea. E-mail: juyoung105@khu.ac. kr; jhkwon@khu.ac.kr

$\dagger$ Electronic supplementary information (ESI) available: Synthesis, experimental, DSC and TGA measurement results, cyclic voltammograms measurement, prompt/delayed PL spectra of DMAC-DPS doped $20 \mathrm{wt} \%$ in each host. ${ }^{1} \mathrm{H}$ NMR and ${ }^{13} \mathrm{C}$ NMR data. See DOI: 10.1039/c8ra01761a

\$ The manuscripts was written through contributions of all authors. All authors have given approval to the final version of the manuscript. These authors contributed equally. (match statement to author names with a symbol).

$\S$ Present addresses: Department of Information Display, Kyung Hee University, 26, Kyungheedae-ro, Dongdaemoon-gu, Seoul, 02447, Republic of Korea. orbital levels, bipolar charge transport ability, and large spectral overlap with doped emitters.

To date, many studies on deep blue TADF OLEDs have been carried out by using unipolar hosts such as $3,3^{\prime}$-bis $(8 a, 9 a-$ dihydro-9H-carbazol-9-yl)-1,1'-biphenyl (mCBP) and 1,3-di(9Hcarbazol-9-yl)benzene ( $\mathbf{m C P})$ are the representative example of hole type hosts. ${ }^{12-14}$ Bis[2-(diphenylphosphino)phenyl] ether oxide (DPEPO) and (2,8-bis(diphenylphosphine oxide)dibenzofuran) (DBFPO) are the most common electron type hosts. ${ }^{12,15,16}$ These phosphine oxide-based hosts have been applied to various deep blue TADF dopant to achieve high efficiency. However, they induce a narrow emission zone due to their unbalanced charge transportation, resulting in high probability of TTA process. In addition, the bonding dissociation energy of their phosphorus-carbon bond is very low. Therefore, the use of phosphine oxide based materials cannot avoid the disadvantages of high driving voltage, serious roll-off phenomena, and short device lifetime. ${ }^{17}$ In order to overcome these issues, several high $T_{1}$ bipolar hosts without phosphine oxide unit have been developed. Most high $T_{1}$ bipolar hosts was mainly based on carbazole as hole transport unit and benzimidazole, ${ }^{18}$ cyano, ${ }^{19}$ and benzimidazobenzothiazole ${ }^{20}$ as electron transport unit. For the first example, Dongge Ma and co-workers reported three bipolar host materials connecting carbazole-based $\mathbf{~ m C P}$ to benzimidazole units by changing the position of a benzimidazole. ${ }^{18}$ The ortho-linked molecule exhibited the highest $T_{1}(3.0 \mathrm{eV})$. In other case, Lian Duan and co-workers synthesized the four host materials, carbazole connecting with cyano units possessed the high $T_{1}$ levels $(2.87-3.03 \mathrm{eV}){ }^{19}$ And finally, Adachi group reported two 


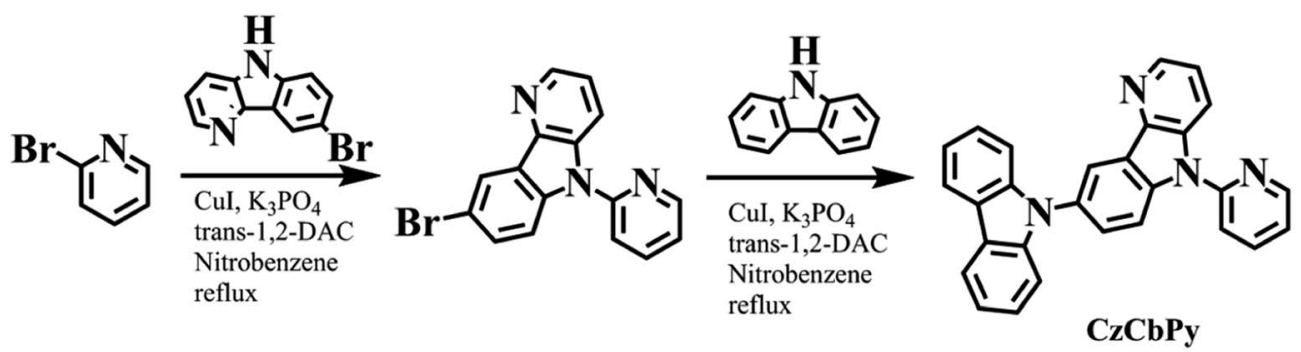

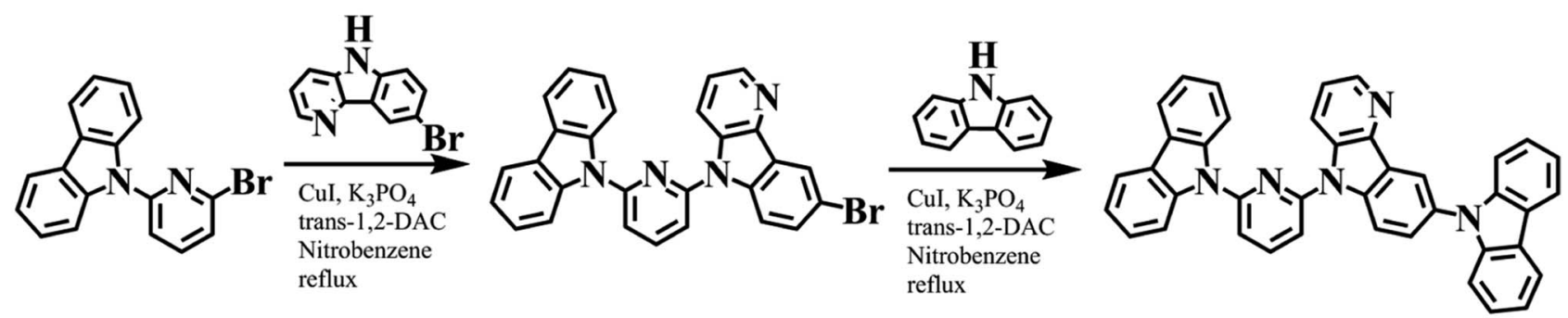

$2 \mathrm{CzCbPy}$

Scheme 1 Synthesis of $\mathrm{CzCbPy}$ and 2CzCbPy.

Table 1 DFT calculation results in energy levels, torsion angles and dipole moment of $\mathrm{CzCbPy}$ and $2 \mathrm{CzCbPy}$

\begin{tabular}{llllllll}
\hline Host & HOMO $(\mathrm{eV})$ & LUMO $(\mathrm{eV})$ & $E_{\mathrm{g}}(\mathrm{eV})$ & $T_{1}(\mathrm{eV})$ & $\theta_{1}\left(^{\circ}\right)$ & $\theta_{2}\left(^{\circ}\right)$ & Dipole moment $(\mu)$ \\
\hline CzCbPy & -5.00 & -1.00 & 4.00 & 3.07 & 50.7 & - & 4.64 \\
2CzCbPy & -5.00 & -1.14 & 3.86 & 3.02 & 50.9 & 39.9
\end{tabular}

\section{Molecular structure}

LUMO

HOMO

\section{$\mathrm{CzCbPy}$}<smiles></smiles>
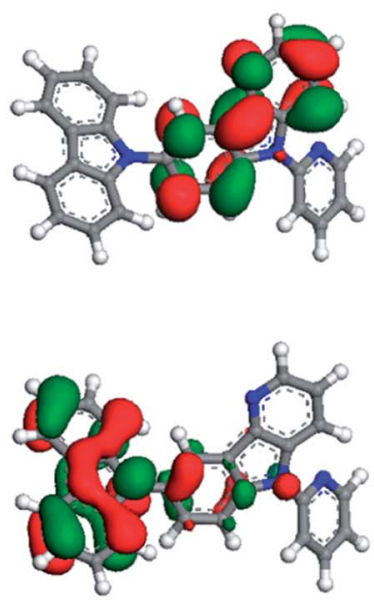

\section{CzCbPy}<smiles></smiles>
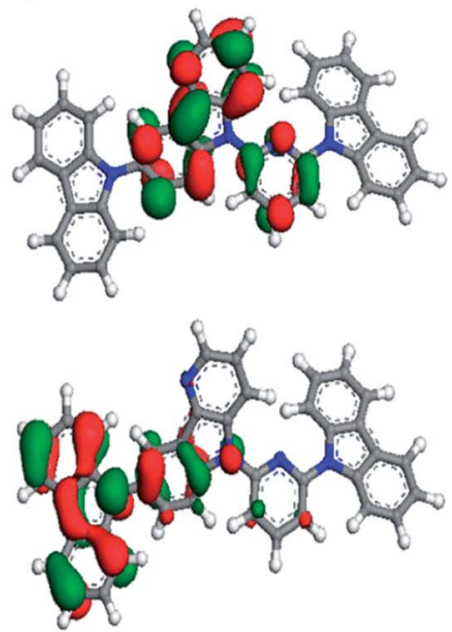

Fig. 1 Molecular structures and HOMO/LUMO distributions of $\mathrm{CzCbPy}$ and $2 \mathrm{CzCbPy}$ in geometry optimization state by DFT simulation with B3LYP/6-31G*. 
Table 2 The photo-physical and electro-physical properties of $\mathrm{CzCbPy}$ and $2 \mathrm{CzCbPy}$

\begin{tabular}{lllllll}
\hline Host & $\lambda_{\mathrm{abs}^{a}}{ }^{a}(\mathrm{~nm})$ & $\lambda_{\mathrm{RTPL}}{ }^{a}(\mathrm{~nm})$ & $E_{\mathrm{g}}{ }^{c}(\mathrm{eV})$ & $\mathrm{HOMO}^{d}(\mathrm{eV})$ & $\mathrm{LUMO}^{e}(\mathrm{eV})$ & $T_{1}{ }^{b}(\mathrm{eV})$ \\
\hline CzCbPy & 286,338 & 355 & 3.50 & 6.12 & 2.62 \\
2CzCbPy & 293,337 & 399 & 3.38 & 6.06 & 2.68 & 2.97
\end{tabular}

${ }^{a}$ Measured in toluene solution of $10^{-5} \mathrm{M}$ at room temperature. ${ }^{b}$ Measured in toluene solution of $10^{-5} \mathrm{M}$ at $77 \mathrm{~K}$. The triplet level was estimated from the first peakt of the phosphorescence spectra. ${ }^{c}$ The optical band gap energy was determined from edge of the absorption spectrum. ${ }^{d}$ HOMO levels were obtained using cyclic voltammetry. ${ }^{e}$ LUMO levels were calculated from optical band gap and HOMO.

(a)

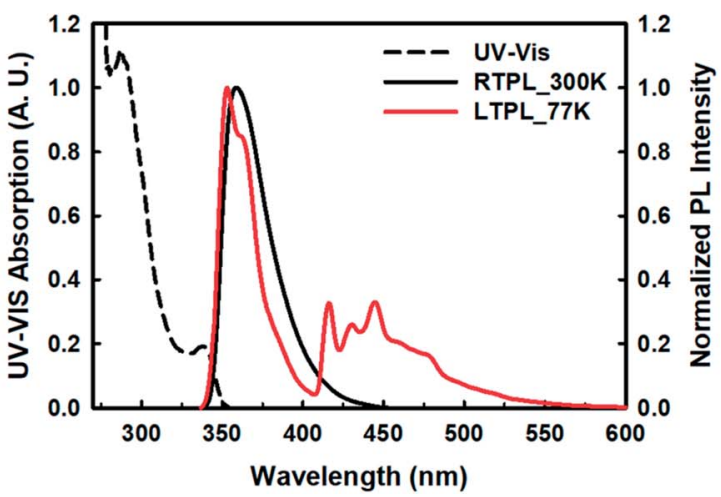

(b)

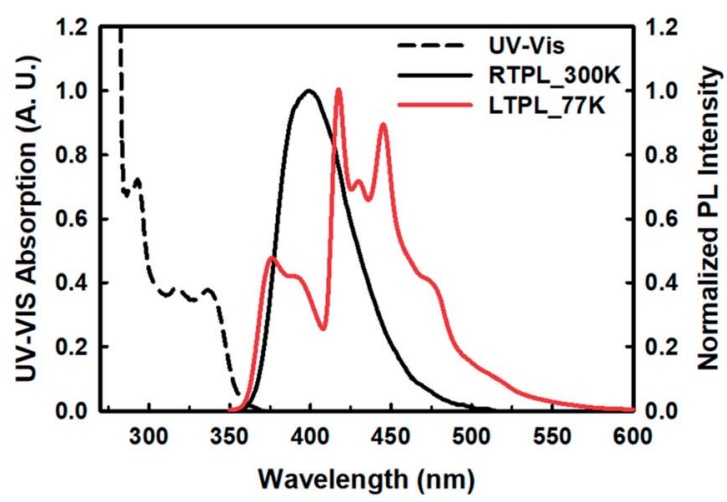

Fig. 2 UV-vis absorption (black dash line), PL spectrum at $300 \mathrm{~K}$ (black solid line) and $77 \mathrm{~K}$ (red solid line) in toluene $10^{-5} \mathrm{M} \mathrm{solution.} \mathrm{(a)} \mathrm{CzCbPy}$, (b) $2 \mathrm{CzCbPy}$.

bipolar hosts connecting benzimidazobenzothiazole and carbazole, and they showed $T_{1}$ value of $3.02-3.04 \mathrm{eV}^{20}$ Even though these materials have been reported, it is necessary to develop new high $T_{1}$ bipolar host for the much better deep blue TADF OLEDs.

In this study, we synthesized 8-(9H-carbazol-9-yl)-5-(pyridin-2-yl)-5H-pyrido[3,2-b]indole (CzCbPy) and 5-(6-(9H-carbazol-9-yl)pyridin-2-yl)-8-(9H-carbazol-9-yl)-5H-pyrido[3,2-b] indole (2CzCbPy) as the high triplet energy bipolar host materials based on carboline and pyridine as electron transport unit and carbazole as hole transport unit. Both CzCbPy and 2CzCbPy hosts showed high $T_{1}$ of about $3.0 \mathrm{eV}$ and high maximum EQE of $22.9 \%$ and low efficiency roll-off characteristic $\left(19.2 \%\right.$ at $\left.1000 \mathrm{~cd} \mathrm{~m}^{-2}\right)$ in TADF blue OLEDs fabricated with bis[4-(9,9-dimethyl-9,10-dihydroacridine) phenyl]sulfone (DMAC-DPS) ${ }^{8}$ as TADF blue dopant. This is the first example of bipolar host without phosphine oxide unit reporting high maximum EQE of above $20 \%$ in DMACDPS based OLED.

\section{Results and discussion}

\section{Material design and synthesis}

Two host materials were designed to possess high triplet energy and bipolar charge transport properties by coupling carbazole as an electron-rich moiety and pyridine as an electron-deficient moiety and $\delta$-carboline as a bipolar moiety. $\delta$-Carboline has high triplet energy than carbazole and bipolar property due to its electron-deficient pyridine unit in the structure. Therefore, carbolines with pyridine unit have been used as an electron transport unit of some high triplet energy host materials. ${ }^{21-23}$ Since carbazole unit is a strong donor and pyridine unit is a weak acceptor, $\delta$-carboline with weak acceptor property was incorporated into the hosts to improve the balance of hole and electron transport property. To keep the high triplet energy of the carbazole and $\delta$-carboline units, all moieties were connected each other through $\mathrm{C}-\mathrm{N}$ bonds because such substitution limits the extension of conjugation. Therefore, it was expected that all

Table 3 Exciton lifetime and the rate constant comparison between $\mathrm{mCP}$ and new host materials

\begin{tabular}{lllllllllll}
\hline Host & $\begin{array}{l}\tau_{\mathrm{P}}{ }^{a} \\
(\mathrm{~ns})\end{array}$ & $\begin{array}{l}\tau_{\mathrm{D}}{ }^{b} \\
(\mu \mathrm{s})\end{array}$ & $\begin{array}{l}\Phi_{\mathrm{PL}}{ }^{c} \\
(\%)\end{array}$ & $\begin{array}{l}\Phi_{\mathrm{P}}{ }^{d} \\
(\%)\end{array}$ & $\begin{array}{l}\Phi_{\mathrm{D}}{ }^{e} \\
(\%)\end{array}$ & $\begin{array}{l}k_{\mathrm{P}} \\
\left(10^{7} \mathrm{~s}^{-1}\right)\end{array}$ & $\begin{array}{l}k_{\mathrm{D}} \\
\left(10^{5} \mathrm{~s}^{-1}\right)\end{array}$ & $\begin{array}{l}k_{\mathrm{r}}^{\mathrm{S}} \\
\left(10^{6} \mathrm{~s}^{-1}\right)\end{array}$ & $\begin{array}{l}k_{\mathrm{ISC}} \\
\left(10^{7} \mathrm{~s}^{-1}\right)\end{array}$ & $\begin{array}{l}k_{\mathrm{RISC}}{ }^{f} \\
\left(10^{6} \mathrm{~s}^{-1}\right)\end{array}$ \\
\hline mCP & 23.88 & 4.03 & 0.67 & 0.17 & 0.50 & 4.17 & 2.48 & 7.08 & 3.46 & 0.879 \\
CzCbPy & 18.83 & 3.10 & 0.76 & 0.16 & 0.60 & 5.31 & 3.23 & 8.50 & 4.46 & 1.44 \\
2CzCbPy & 20.59 & 3.29 & 0.72 & 0.16 & 0.56 & 4.86 & 3.04 & 7.77 & 4.08 & 1.27
\end{tabular}

${ }^{a}$ Exciton lifetime of prompt components of $20 \%$ doped film with DMAC-DPS. ${ }^{b}$ Exciton lifetime of delayed components of $20 \%$ doped film with DMAC-DPS. ${ }^{c}$ PLQY measured with integral sphere. ${ }^{d}$ Divided prompt component from PLQY. ${ }^{e}$ Divided delayed component from PLQY. ${ }^{f}$ Rate constant of reverse intersystem crossing from $T_{1}$ to $\mathrm{S}_{1} \cdot{ }^{g}$ Rate constant of non-radiative decay from $T_{1}$ to ground state $\left(\mathrm{S}_{0}\right)$. 


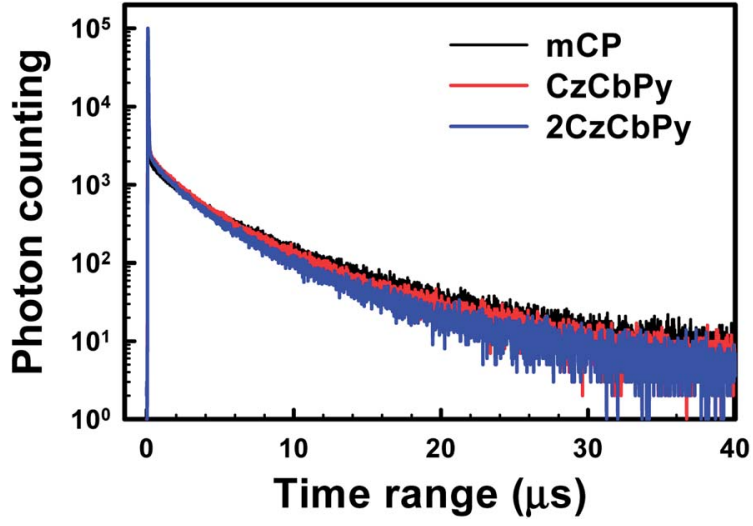

Fig. 3 Transient $\mathrm{PL}$ decay curves of $\mathrm{mCP}, \mathrm{CzCbPy}$, and $2 \mathrm{CzCbPy}$ films doped with 20 wt\% DMAC-DPS. hosts could show high triplet energy and bipolar charge transport properties. Two host materials, $\mathbf{C z C b P y}$ and $2 \mathrm{CzCbPy}$, were prepared by two copper-catalyzed Ullmann couplings of halogenated compounds with amines (Scheme 1) and purified successively by column chromatography and vacuum train sublimation yielding highly pure compounds with a purity above $99 \%$. The chemical structures were characterized by ${ }^{1} \mathrm{H}$ and ${ }^{13} \mathrm{C}$ NMR and high resolution mass spectrometer (HRMS).

\section{Theoretical calculations}

A molecular simulation was carried out to understand the physical properties of $\mathbf{C z C b P y}$ and $\mathbf{2 C z C b P y}$ at the molecular level (Table 1). Theoretical calculations were performed using density functional theory (DFT) calculation with B3LYP in Dmol3. The optimized structures and the HOMO and LUMO orbitals for CzCbPy and 2CzCbPy are shown in Fig. 1. The HOMOs of both hosts are mainly distributed over the carbazole

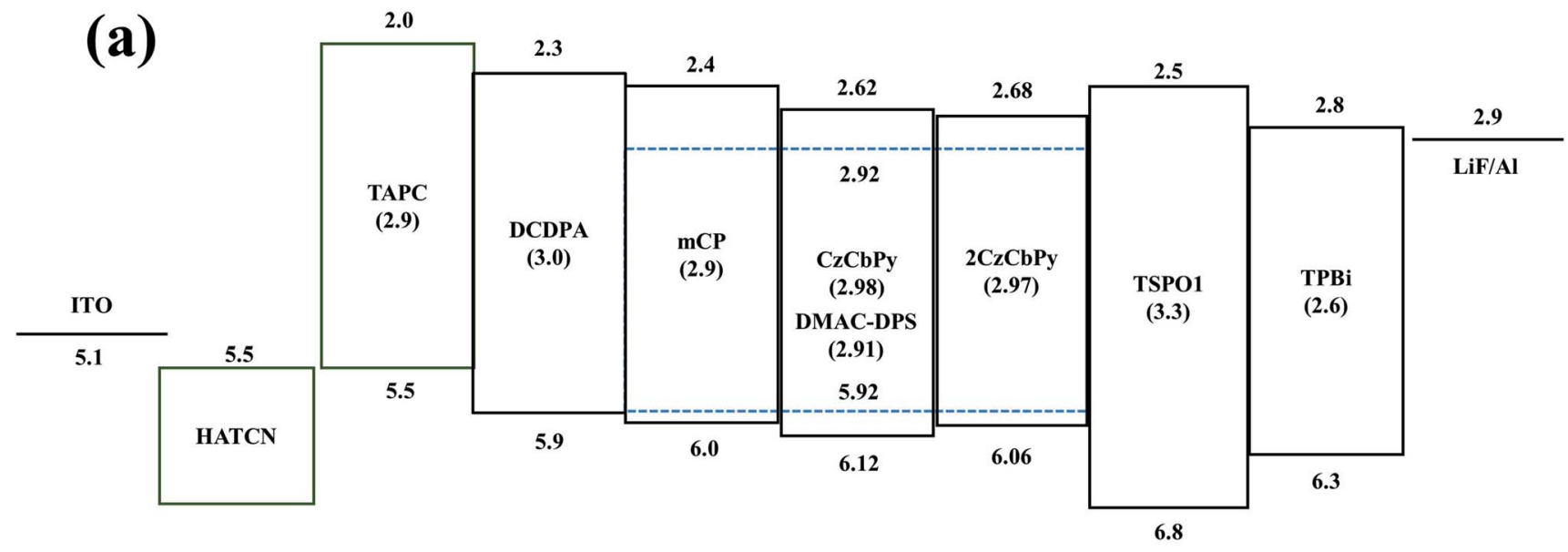

(b)<smiles>N#Cc1nc2c3nc(C#N)c(C#N)nc3c3nc(C#N)c(C#N)nc3c2nc1C#N</smiles>

HATCN<smiles></smiles>

DMAC-DPS<smiles>Cc1ccc(N(c2ccc(C)cc2)c2ccc(C3(c4ccc(N(c5ccc(C)cc5)c5ccc(C)cc5)cc4)CCCCC3)cc2)cc1</smiles>

TAPC<smiles>O=P(c1ccccc1)(c1ccccc1)c1ccc([Si](c2ccccc2)(c2ccccc2)c2ccccc2)cc1</smiles>

TSPO1<smiles>c1ccc(N(c2ccccc2)c2cc(-n3c4ccccc4c4ccccc43)cc(-n3c4ccccc4c4ccccc43)c2)cc1</smiles>

\section{DCDPA}<smiles>c1ccc(-n2c(-c3cc(-c4nc5ccccc5n4-c4ccccc4)cc(-c4nc5ccccc5n4-c4ccccc4)c3)nc3ccccc32)cc1</smiles>

TPBi

Fig. 4 (a) Diagram of the evaluated device structure and energy level of the device. (b) The molecular structures of material for each layer. 
(a)

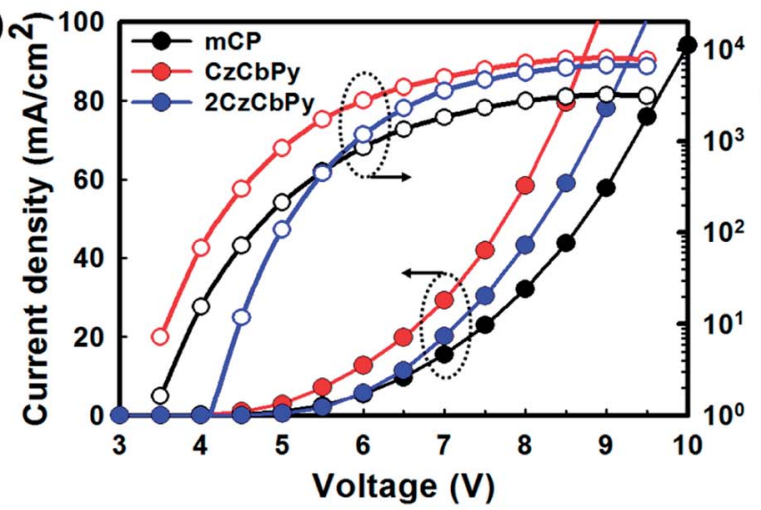

(c)

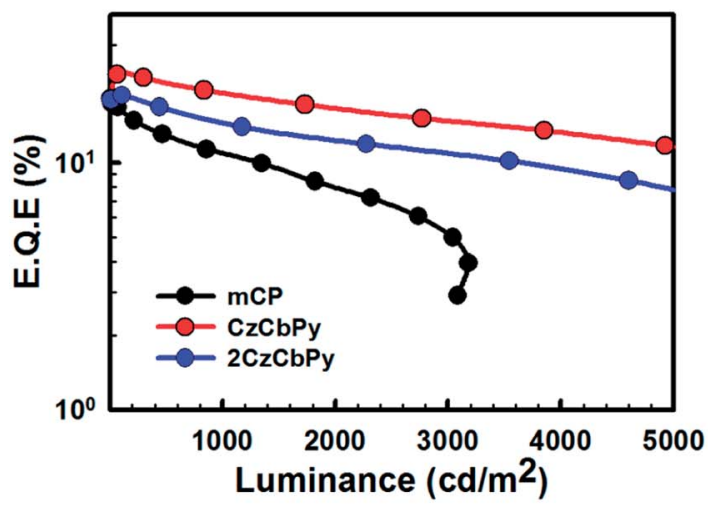

(b)

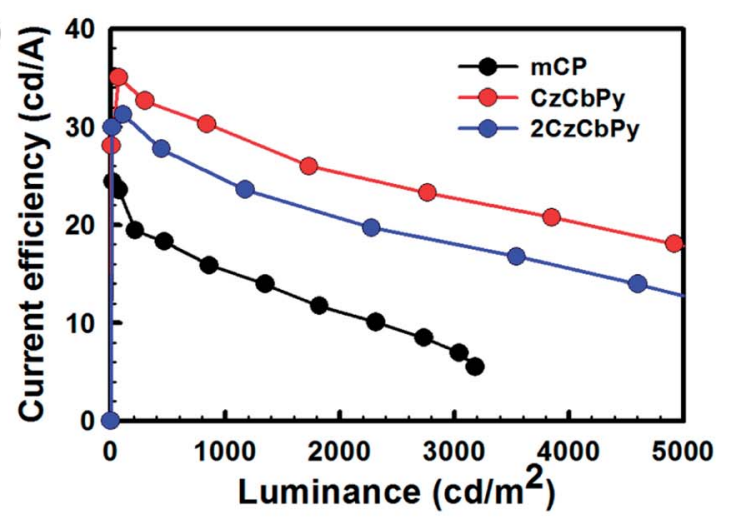

(d)

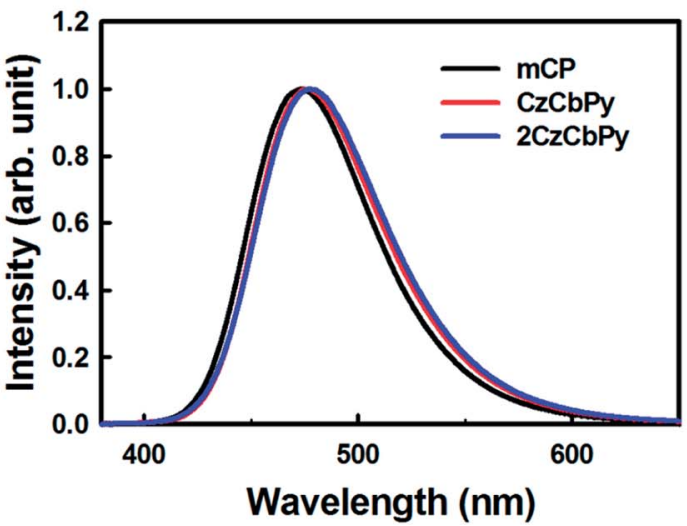

Fig. 5 (a) J-V-L characteristics (b) CE-L curves, (c) EQE-L curves, and (d) EL spectra of blue TADF-OLEDs using mCP, CzCbPy and $2 C z C b P y$ with DMAC-DPS.

near the $\delta$-carboline side and partly the $\delta$-carboline. Therefore, the HOMO levels of these hosts are same as $-5.0 \mathrm{eV}$. On the other hand, the LUMO orbitals of these hosts are differently dispersed: the LUMO of CzCbPy is only observed in the $\delta$-carboline, while the LUMO of $2 \mathbf{C z C b P y}$ is localized not only on the $\delta$-carboline but also on the pyridine. As a result, the LUMO levels of two hosts are different from each other.

The LUMO level of $2 \mathrm{CzCbPy}$ is $-1.4 \mathrm{eV}$, which is $0.14 \mathrm{eV}$ lower than that of $\mathbf{C z C b P y}$, implying that the additional carbazole in $2 \mathrm{CzCbPy}$ reduced the LUMO level of $2 \mathbf{C z C b P y}$. The HOMOs of both host materials were formed at carbazole moiety and the LUMOs were formed at carboline and pyridine moieties. Since the HOMO is in hole transport type moiety and LUMO is in electron transport type moieties, bipolar transport property of our two hosts was expected. In addition, these hosts also show well separated HOMO and LUMO orbitals, which could result in bipolar characteristic. The calculated triplet energy levels of CzCbPy and $\mathbf{2 C z C b P y}$ were $3.07 \mathrm{eV}$ and $3.02 \mathrm{eV}$, respectively. The $T_{1}$ of $\mathbf{C z C b P y}$ is slightly higher than that of 2CzCbPy. To understand the reason of this difference, we investigated the torsion angle between carbazole, $\delta$-carboline, and pyridine. The angles $\left(\theta_{1}\right)$ between carbazole and carboline for $\mathbf{C z C b P y}$ and $2 \mathrm{CzCbPy}$ were similar and they were $50.5^{\circ}$ and $50.9^{\circ}$, respectively, which are large enough to make two moieties well separated. However, 2CzCbPy has additional carbazole and the angle $\left(\theta_{2}\right)$ between carbazole and pyridine is $39.9^{\circ}$, which is small and makes the conjugation length longer, resulting in lower $T_{1}$. In addition, the molecular dipole moments of two hosts were calculated at ground states. The dipole moment of CzCbPy is $4.64 \mu$, which is slightly higher than that of $2 \mathrm{CzCbPy}(4.60 \mu)$, due to the greater asymmetry of CzCbPy. From the molecular dipole moment, we speculated that $\mathbf{C z C b P y}$ would make slightly higher polar environment than 2CzCbPy.

\section{Material properties}

The thermal properties of $\mathbf{C z C b P y}$ and $\mathbf{2 C z C b P y}$ were examined by differential scanning calorimetry (DSC) and thermogravimetric analysis (TGA) and their thermograms are shown in Fig. S1 $\dagger$ and summarized in Table $\mathrm{S} 1 . \dagger$ The glass transition temperature $\left(T_{\mathrm{g}}\right)$ of $2 \mathbf{C z C b P y}\left(138^{\circ} \mathrm{C}\right)$ is higher than that of CzCbPy $\left(81^{\circ} \mathrm{C}\right)$. These values are higher than that of the wellknown blue host material, 1,3-bis( $N$-carbazolyl)benzene

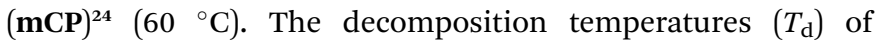
CzCbPy and 2CzCbPy were $321{ }^{\circ} \mathrm{C}$ and $412{ }^{\circ} \mathrm{C}$, respectively. Overall, 2CzCbPy showed much higher thermal stability than CzCbPy, due to the higher molecular weight by additional carbazole moiety. Photophysical properties of CzCbPy and 2CzCbPy were examined using UV-vis and photoluminescence (PL) spectroscopies. Fig. 2 shows UV-vis absorption and PL spectra of CzCbPy and 2CzCbPy. The CzCbPy exhibited absorption peaks at $286,338 \mathrm{~nm}$, and $2 \mathrm{CzCbPy}$ indicated at 293, $337 \mathrm{~nm}$, respectively. Both peaks of 338 and $337 \mathrm{~nm}$ are 
(a)

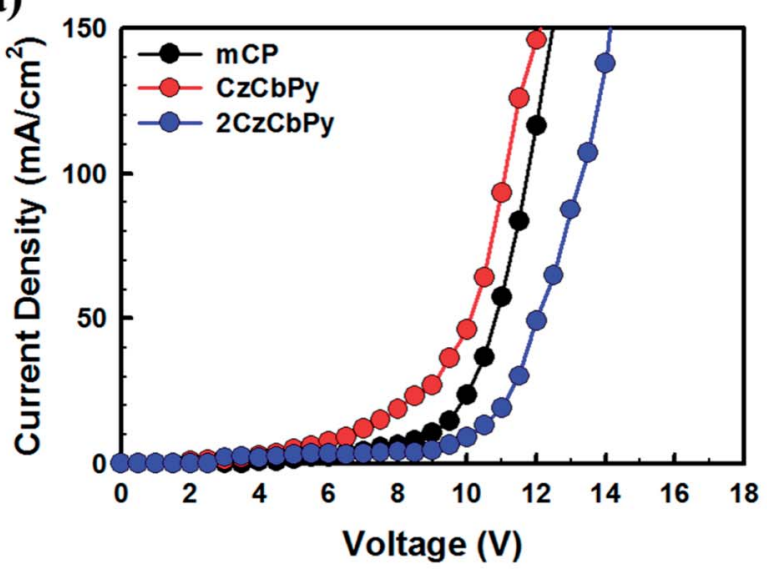

(b)

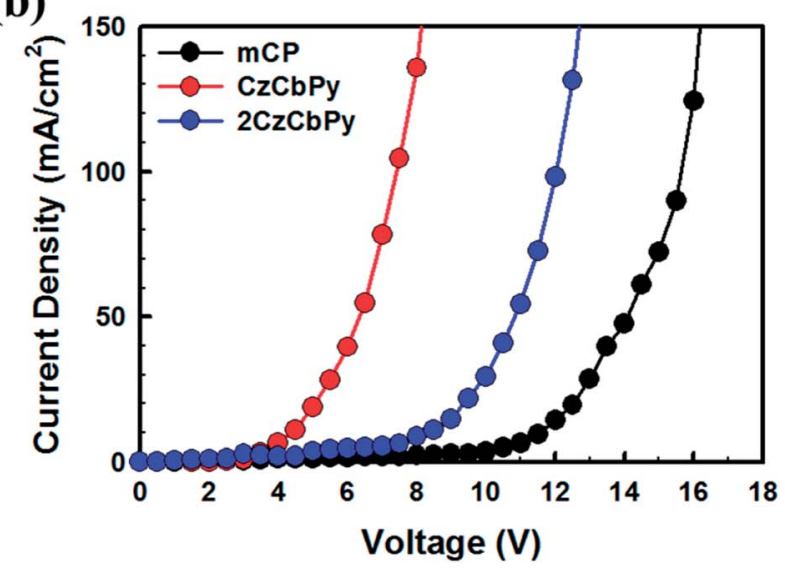

Fig. $6 \mathrm{~J}-V$ characteristics of (a) HOD and (b) EOD of mCP, $\mathrm{CzCbPy}$, and $2 \mathrm{CzCbPy}$.

assigned to the $\pi-\pi^{*}$ transition of $\mathbf{C z C b P y}$ and $2 \mathrm{CzCbPy}$, respectively. The band gap energy $\left(E_{\mathrm{g}}\right)$ of $\mathbf{C z C b P y}$ and $2 \mathrm{CzCbPy}$ calculated from the absorption edge of UV-vis spectra are $3.50 \mathrm{eV}$ and $3.38 \mathrm{eV}$, respectively. The band gap of $\mathbf{C z C b P y}$ is $0.12 \mathrm{eV}$ wider than $\mathbf{2 C z C b P y}$, which is similar to the simulation results. The phosphorescence spectra of CzCbPy and $2 \mathbf{C z C b P y}$ revealed their $T_{1}$ as $\sim 3.0 \mathrm{eV}$ for both TADF hosts. Thus, $T_{1}$ of both hosts are higher than that of common carbazole-based host, MCP $(2.9 \mathrm{eV})^{25}$ and high enough for application in deep blue TADF OLEDs. The HOMO levels of CzCbPy and 2CzCbPy measured by cyclic voltammetry (Fig. S2 $\dagger$ ) are $-6.12 \mathrm{eV}$ and $-6.06 \mathrm{eV}$, respectively. LUMO levels of $\mathbf{C z C b P y}(-2.62 \mathrm{eV})$ and 2CzCbPy $(-2.68 \mathrm{eV})$ are estimated from $E_{\mathrm{g}}$ and HOMO levels. Measured HOMO and LUMO values of both hosts have similar tendency with molecular simulation data even though absolute values are different due to approximate quantum mechanics calculation. The HOMO levels of these two hosts are similar to that of $\mathbf{~ m C P}(-5.9 \mathrm{eV})$, whereas the LUMO levels are much deeper than that of $\mathbf{m C P}(-2.4 \mathrm{eV})$, due to the additional electron acceptor moieties (Table 2).

\section{Photophysical properties}

To investigate the applicability of two hosts in TADF OLED, 20\% doped films of DMAC-DPS in the hosts and $\mathbf{~ M C P}$ were prepared.
The prompt and delayed PL spectra were similar for all hosts (Fig. S3†). This indicates that the spectra originated from only DMAC-DPS and the energy of these hosts is well transferred to the dopant. The prompt PL spectrum in mCP film was $466 \mathrm{~nm}$, and those in CzCbPy and 2CzCbPy were $474 \mathrm{~nm}$. Since CzCbPy and $2 \mathrm{CzCbPy}$ have higher dipole moment and create more polar condition for DMAC-DPS than $\mathbf{M C P}$, the CT state $\left(\mathrm{S}_{1}\right)$ of DMACDPS was more stabilized in $\mathbf{C z C b P y}$ and $2 \mathrm{CzCbPy}$, resulting in the red-shift of the spectra. This result implies that the emission characteristics of the dopant may be varied with the host. As shown in Fig. 3, the transient PL decay curve of the films measured under nitrogen atmosphere clearly exhibits both a nanosecond-scale prompt component and a micro-secondscale delayed component at room temperature $(300 \mathrm{~K})$. The prompt lifetimes were similar in all three hosts, about $20 \mathrm{~ns}$, but the delayed exciton lifetime was shorter in CzCbPy and $2 \mathbf{C z C b P y}$ (3.10 and $3.29 \mu \mathrm{s}$, respectively) than in $\mathbf{m C P}(4.03 \mu \mathrm{s})$. As mentioned above, $\mathrm{S}_{1}$ of the dopant is more stabilized in polar CzCbPy and $2 \mathrm{CzCbPy}$, which leads to the decrease of $\Delta E_{\mathrm{ST}}$ and short delayed exciton lifetime. The overall PL quantum yields (PLQYs) were 67.32, 75.90, and 72.19\% for $\mathbf{m C P}, \mathbf{C z C b P y}$, and 2CzCbPy, respectively (Table 3). The prompt components of PLQYs in mCP, CzCbPy, and 2CzCbPy were similar, 17.17, 15.86 , and $15.59 \%$, and the delayed ones were different, $50.15 \%$, $60.04 \%$, and 56.60\%, respectively. The delayed PLQYs are much larger in $\mathbf{C z C b P y}$ and $\mathbf{2 C z C b P y}$ than in $\mathbf{~ m C P}$, which means the RISC process in CzCbPy and $\mathbf{2 C z C b P y}$ is more efficient than in mCP. To know the exact RISC efficiency, the rate constant of RISC $\left(k_{\text {RISC }}\right)$ was calculated using the reported method. ${ }^{26}$ The RISC rate is much faster $\left(1.44 \times 10^{6} \mathrm{~s}^{-1}, 1.27 \times 10^{6} \mathrm{~s}^{-1}\right)$ in CzCbPy and $2 \mathbf{C z C b P y}$ than in $\mathbf{m C P}\left(8.79 \times 10^{5} \mathrm{~s}^{-1}\right)$. Therefore, it is expected that the TADF characteristic of DMAC-DPS will be better in these two hosts.

\section{Device performances}

To evaluate the performances of $\mathbf{C z C b P y}$ and $2 \mathrm{CzCbPy}$ as host materials for TADF-OLEDs, deep blue TADF-OLEDs with DMACDPS were fabricated. The device based on $\mathbf{~ M C P}$ was also fabricated as a reference. The device structures were ITO/ 1,4,5,8,9,11-hexaazatriphenylene-hexacarbonitrile (HATCN, 7 $\mathrm{nm}) / 4,4^{\prime}$-cyclohexylidenebis[ $N, N$-bis(4-methylphenyl)

benzenamine] (TAPC, $40 \mathrm{~nm}) / 3,5-\mathrm{di}(9 H$-carbazol-9-yl)- $N, N$ diphenylaniline (DCDPA, $10 \mathrm{~nm}$ )/host: $20 \mathrm{wt} \%$ DMAC-DPS (25 $\mathrm{nm}$ )/diphenyl(4-(triphenylsilyl)phenyl)phosphine oxide (TSPO1, $5 \mathrm{~nm}) / 2,2^{\prime}, 2^{\prime \prime}$-(1,3,5-benzinetriyl)-tris(1-phenyl-1- $H$-benzimidazole) (TPBi, $30 \mathrm{~nm}) / \mathrm{LiF}(1.5 \mathrm{~nm}) / \mathrm{Al}$ (100 nm) (Fig. 4). Fig. 5a shows the current density-voltage-luminance curves of the deep blue TADF-OLEDs. The CzCbPy device showed higher current density than the $\mathbf{2 C z C b P y}$ device. In addition, both devices showed higher current density than the mCP device due to their bipolar charge transport properties. The luminance was also higher in CzCbPy device (8035 $\mathrm{cd} \mathrm{m}^{-2}$ ) than 2CzCbPy and $\mathbf{m C P}$ (6624 $\mathrm{cd} \mathrm{m}^{-2}$ and $3181 \mathrm{~cd} \mathrm{~m}^{-2}$, respectively) due to the short delayed exciton lifetime and broad exciton recombination zone derived from bipolar characteristic. The current efficiencies of the $\mathbf{C z C b P y}$ and $2 \mathbf{C z C b P y}$ devices were much higher than that of 
(a)

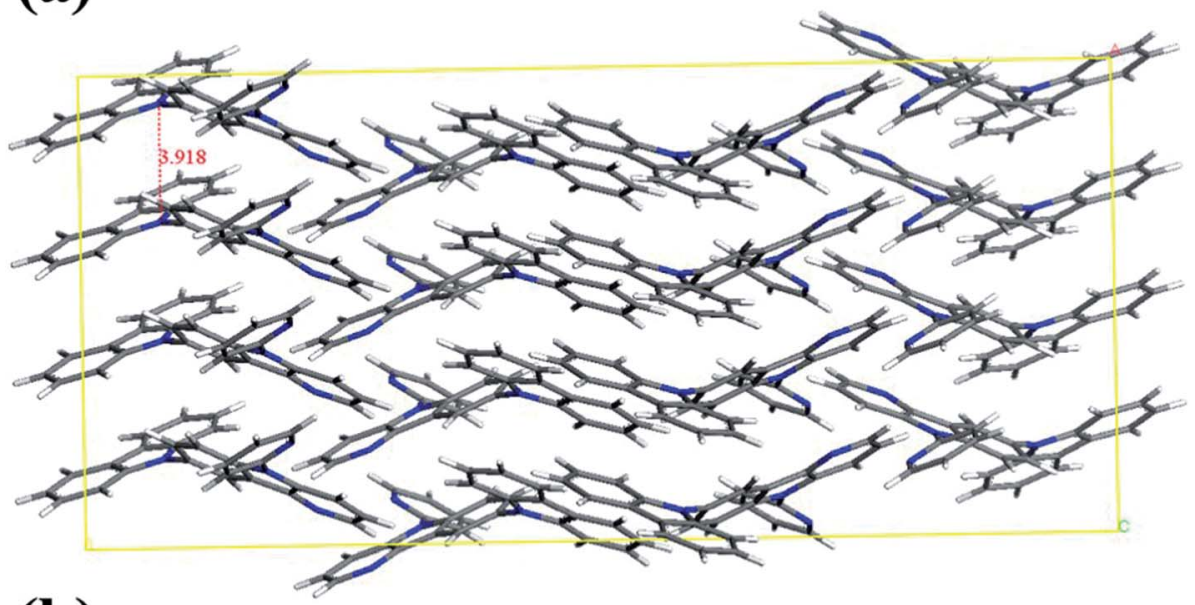

(b)

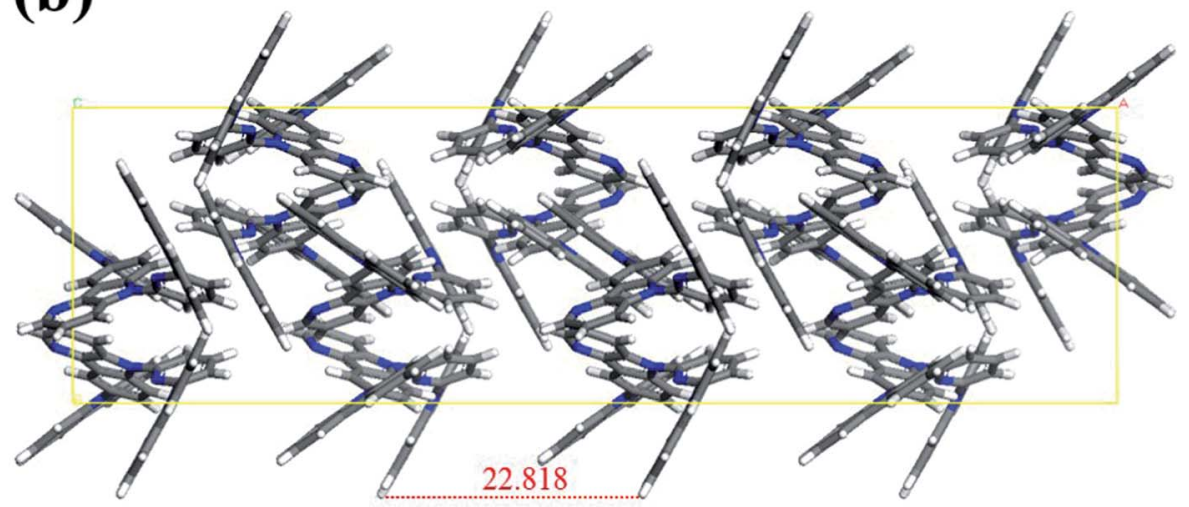

Fig. 7 Molecular packing simulation results of (a) $\mathrm{CzCbPy}$ and (b) $2 \mathrm{CzCbPy}$.

mCP device (35.0 cd A $\mathrm{ct}^{-1}$ and $31.3 \mathrm{~cd} \mathrm{~A}^{-1}$ vs. $24.4 \mathrm{~cd} \mathrm{~A}^{-1}$ ) as shown in Fig. $5 \mathrm{~b}$. The maximum external quantum efficiency (EQE) of CzCbPy device was $22.9 \%$ as shown in Fig. 5 c, which is higher than those of 2CzCbPy and MCP devices $(18.8 \%$ and $17.5 \%$, respectively) and one of the highest efficiency reported so far for deep blue TADF OLED using phosphine oxide-free host. In addition, the CzCbPy device exhibited high EQE of $19.2 \%$ even at $1000 \mathrm{~cd} \mathrm{~m}^{-2}$, which showed lower efficiency roll- off $(16 \%)$ than those of 2 CzCbPy and $\mathbf{m C P}$ devices (23\% and $38 \%$, respectively). Such high EQE and low efficiency roll-off of CzCbPy device are due to its higher PLQY (75.90\%) and faster RISC rate $\left(1.44 \times 10^{6} \mathrm{~s}^{-1}\right)$ than those of $2 \mathrm{CzCbPy}$ and $\mathbf{m C P}$ devices $\left(72.19 \%\right.$ and $1.27 \times 10^{6} \mathrm{~s}^{-1}, 67.32 \%$ and $8.79 \times 10^{5} \mathrm{~s}^{-1}$, respectively). The faster RISC and bipolar characteristics of CzCbPy and $2 \mathrm{CzCbPy}$ reduce triplet-triplet annihilation and exciton-polaron annihilation by transporting holes and

Table 4 Electroluminescence properties of the TADF-OLEDs with new host materials

\begin{tabular}{|c|c|c|c|c|c|c|c|c|c|}
\hline \multirow[b]{2}{*}{ Hosts } & \multirow[b]{2}{*}{$\begin{array}{l}\text { Voltage }^{a} \\
{[\mathrm{~V}]}\end{array}$} & \multirow[b]{2}{*}{$\begin{array}{l}\text { Voltage }^{b} \\
{[\mathrm{~V}]}\end{array}$} & \multicolumn{4}{|c|}{ Maximum efficiency at $1000 \mathrm{~cd} \mathrm{~m}^{-2}$} & \multirow[b]{2}{*}{$\begin{array}{l}\text { Efficiency } \\
\text { roll-off }^{e}[\%]\end{array}$} & \multirow[b]{2}{*}{$\begin{array}{l}\text { Max luminance } \\
{\left[\mathrm{cd} \mathrm{m}^{-2}\right]}\end{array}$} & \multirow[b]{2}{*}{$\begin{array}{l}\operatorname{CIE}^{f} \\
{[x, y]}\end{array}$} \\
\hline & & & $\begin{array}{l}\mathrm{CE}^{c} \\
{\left[\mathrm{~cd} \mathrm{~A}^{-1}\right]}\end{array}$ & $\begin{array}{l}\mathrm{EQE}^{d} \\
{[\%]}\end{array}$ & $\begin{array}{l}\mathrm{CE}^{c} \\
{\left[\mathrm{~cd} \mathrm{~A}^{-1}\right]}\end{array}$ & $\begin{array}{l}\mathrm{EQE}^{d} \\
{[\%]}\end{array}$ & & & \\
\hline mCP & 3.3 & 6.1 & 24.4 & 17.5 & 15.2 & 10.8 & 38.3 & 3181 & $\begin{array}{l}(0.16, \\
0.23)\end{array}$ \\
\hline CzCbPy & 3.0 & 5.1 & 35.0 & 22.9 & 29.7 & 19.8 & 16.2 & 8035 & $\begin{array}{l}(0.15, \\
0.26)\end{array}$ \\
\hline 2CzCbPy & 4.0 & 5.9 & 31.3 & 18.8 & 24.6 & 14.5 & 22.9 & 6624 & $\begin{array}{l}(0.16, \\
0.28)\end{array}$ \\
\hline
\end{tabular}

${ }_{f}^{a}$ Measured at $1 \mathrm{~cd} \mathrm{~m}^{-2} \cdot{ }^{b}$ Measured at $1000 \mathrm{~cd} \mathrm{~m}^{-2} \cdot{ }^{c}$ Current efficiency. ${ }^{d}$ External quantum efficiency. ${ }^{e}$ Ratio from maximum EQE at $1000 \mathrm{~cd} \mathrm{~m}{ }^{-2}$.

${ }^{f}$ Measured at $10 \mathrm{~mA} \mathrm{~cm}{ }^{-2}$. 
Table 5 The length of each corner of the cell packed 16 molecules and calculating results of volume and density

\begin{tabular}{llllllr}
\hline Host & $A(\AA)^{a}$ & $B(\AA)^{a}$ & $C(\AA)^{a}$ & Cell volume & ${\left.\text { Density }(\mathrm{g} \mathrm{cm})^{-3}\right)}^{\text {Distance }(\AA)^{b}}$ \\
\hline CzCbPy & 15.67 & 34.14 & 15.19 & 8126.25 & 1.34 & 3.918 \\
2CzCbPy & 45.63 & 12.92 & 20.43 & 12044.29 & 1.26
\end{tabular}

${ }^{a}$ The length of each cell in the $x, y$, and $z$ axis directions. ${ }^{b}$ The distance between two parallel molecules.

electrons to the dopant rapidly and efficiently compared with mCP. As a result, the efficiency roll-off characteristics of $\mathbf{C z C b P y}$ and $2 \mathrm{CzCbPy}$ devices are better than that of $\mathbf{m C P}$ device. To examine the bipolar properties of the two hosts, hole only device (HOD) and electron only device (EOD) with the structures ITO/ TAPC $(20 \mathrm{~nm}) /$ host $(50 \mathrm{~nm}) / \mathbf{T A P C}(20 \mathrm{~nm}) / \mathrm{Al}(100 \mathrm{~nm})$ and ITO/ TmPyPB $(20 \mathrm{~nm}) /$ host $(50 \mathrm{~nm}) / \mathbf{T P B i}(20 \mathrm{~nm}) / \mathrm{LiF}(1.5 \mathrm{~nm}) / \mathrm{Al}$ (100 $\mathrm{nm}$ ), respectively, were fabricated, using common host $\mathbf{~} \mathbf{C P}$ as a reference. The current densities of $50 \mathrm{~mA} \mathrm{~cm}^{-2}$ in the HODs of mCP, CzCbPy, and 2CzCbPy were measured at $10.8 \mathrm{~V}, 10.1 \mathrm{~V}$, and $13.5 \mathrm{~V}$, respectively, and it was measured in EOD as $14 \mathrm{~V}$, $6.4 \mathrm{~V}$, and $10.8 \mathrm{~V}$, respectively, as shown in Fig. 6. Here the hole current density of HODs with CzCbPy is slightly higher than that of $\mathbf{~ m C P}$, while that of $\mathbf{2 C z C b P y}$ is much lower than those of other hosts. However, in the EODs, all hosts exhibited different electron current density. The CzCbPy shows the highest electron current density, and then $2 \mathrm{CzCbPy}$, followed by $\mathbf{m C P}$. The additional carboline and pyridine units in $\mathbf{C z C b P y}$ and $2 \mathrm{CzCbPy}$ improved the electron transport property of the hosts. Hence, CzCbPy has the best charge transport property, which can explain that CzCbPy device showed higher efficiency and lower roll-off than 2CzCbPy and $\mathbf{m C P}$ devices. By the way, CzCbPy has one less carbazole unit than $\mathbf{2} \mathbf{C z C b P y}$ and it was expected that the hole current density of $\mathbf{C z C b P y}$ would be less than that of 2CzCbPy. To look into the cause of the better charge transport property of $\mathbf{C z C b P y}$, the molecular packing structures of each host were analysed using polymorph simulation (Fig. 7 and Table 5). The 16 molecules of CzCbPy and 2CzCbPy in packed structure with the lowest energy were selected and the distance between the molecules and the density of each cell were compared. The distance between molecules of CzCbPy (3.92 ̊) is closer than that of $2 \mathrm{CzCbPy}(22.82 \AA)$, which means the order of the molecules and the density of each cell in the former $\left(1.34 \mathrm{~g} \mathrm{~cm}^{-3}\right)$ is much higher than the later $\left(1.27 \mathrm{~g} \mathrm{~cm}^{-3}\right)$. Indeed, as shown in the Fig. 7a, the molecules are well aligned in case of $\mathbf{C z C b P y}$, whereas such type of arrangement is not observed in case of $2 \mathrm{CzCbPy}$ (Fig. 7b). This type of molecular arrangement in CzCbPy makes the intermolecular $\pi-\pi$ interactions better than in $2 \mathbf{C z C b P y}$, thus faster hole and electron mobility observed in $\mathbf{C z C b P y}$ than $2 \mathrm{CzCbPy}{ }^{27}$ It indicates that CzCbPy has the best charge balance so that the exciton recombination occurs well, resulting in higher efficiency.

Electroluminescence (EL) spectra and the color coordinates of all devices were shown in Fig. 5d and Table 4. The CzCbPy and $2 \mathrm{CzCbPy}$ devices showed slightly red-shifted spectra compared to $\mathbf{~ m C P}$ device. The color coordinates were $(0.15$, 0.26), (0.16, 0.28), and (0.16, 0.23) for CzCbPy, 2CzCbPy, and
mCP devices, respectively. These red-shifts of the color coordinates can be ascribed to the higher polarities of the two hosts compared with $\mathbf{~ M C P}$ and the shift of the recombination zone from the electron transport layer side to the hole transport layer side due to the increased electron mobility of $\mathbf{C z C b P y}$ and 2CzCbPy. CzCbPy and 2CzCbPy have high polarity due to their donor-acceptor structures, which serve polar medium to the dopant, resulting in the stabilization of $\mathrm{S}_{1}$ and the red shift of the spectra as shown in Fig. S4.†

\section{Conclusions}

In summary, two $\delta$-carboline-based, phosphine oxide-free host materials were prepared for deep blue TADF OLEDs. The CzCbPy and 2CzCbPy have the same high triplet energy of $3.05 \mathrm{eV}$ and bipolar charge-transport properties. The CzCbPy device doped with DMAC-DPS showed a high quantum efficiency of $22.9 \%$ and a small efficiency roll-off of $16 \%$. Such efficiency is one of the highest reported so far in the deep blue TADF OLEDs using phosphine oxide-free host and comparable to that of OLEDs using phosphine oxide based hosts. Evaluation of the lifetime of CzCbPy based device is in progress.

\section{Conflicts of interest}

There are no conflicts to declare.

\section{Acknowledgements}

This work was supported by Grant No. NRF2016R1A6A3A11930666 and by the Human Resources Development program (no. 20154010200830) of the Korea Institute of Energy Technology Evaluation and Planning (KETEP) grant funded by the Korea Government Ministry of Trade, Industry and Energy. And this work was also supported by the Industrial Strategic Technology Development Program of MKE/KEIT (10048317).

\section{References}

1 C. Adachi, Third-generation organic electroluminescence materials, J. Appl. Phys., 2014, 53, 060101.

2 H. Xu, R. Chen, Q. Sun, W. Lai, Q. Su, W. Huang and X. Liu, Recent progress in metal-organic complexes for optoelectronic applications, Chem. Soc. Rev., 2014, 43, 3259-3302.

3 Y. Tao, K. Yuan, T. Chen, P. Xu, H. Li, R. Chen, C. Zheng, L. Zhang and W. Huang, Thermally Activated Delayed 
Fluorescence Materials Towards the Breakthrough of Organoelectronics, Adv. Mater., 2014, 26, 7931-7958.

$4 \mathrm{X}$. Yang, G. Zhou and W. Y. Wong, Functionalization of phosphorescent emitters and their host materials by maingroup elements for phosphorescent organic light-emitting devices, Chem. Soc. Rev., 2015, 44, 8484-8575.

5 S. Reineke, Organic light-emitting diodes: Phosphorescence meets its match, Nat. Photonics, 2014, 8, 269-270.

6 T. T. Bui, F. Goubard, M. I. Ouali, D. Gigmes and F. Dumur, Recent advances on organic blue thermally activated delayed fluorescence emitters for organic light-emitting diodes, Beilstein J. Org. Chem., 2018, 14, 282-308.

7 H. Uoyama, K. Goushi, K. Shizu, H. Nomura and C. Adachi, Highly efficient organic light-emitting diodes from delayed fluorescence, Nature, 2012, 492, 234-238.

8 Q. Zhang, B. Li, S. Huang, H. Nomura, H. Tanaka and C. Adachi, Efficient blue organic light-emitting diodes employing thermally activated delayed fluorescence, Nat. Photonics, 2014, 8, 326-332.

9 S. Y. Kim, W. I. Jeong, C. Mayr, Y. S. Park, K. H. Kim, J. H. Lee, C. K. Moon, W. Bruttig and J. J. Kim, Organic Light-Emitting Diodes with 30\% External Quantum Efficiency Based on a Horizontally Oriented Emitter, Adv. Funct. Mater., 2013, 23, 3896-3900.

10 K. C. Pan, S. W. Li, Y. Y. Ho, Y. J. Shiu, W. L. Tsai, M. Jiao, W. K. Lee, C. C. Wu, C. L. Chung, T. Chatterjee, Y. S. Li, K. T. Wong, H. C. Hu, C. C. Chen and M. T. Lee, Efficient, and Tunable Thermally Activated Delayed Fluorescence Emitters Having Orientation Adjustable CN-Substituted Pyridine and Pyrimidine Acceptor Units, Adv. Funct. Mater., 2016, 42, 7560-7571.

11 H. Kaji, H. Suzuki, T. Fukushima, K. Shizu, K. Suzuki, S. Kubo, T. Komino, H. Oiwa, F. Suzuki, A. Wakamiya, Y. Murata and C. Adachi, Purely organic electroluminescent material realizing 100\% conversion from electricity to light, Nature, 2015, 6, 8476.

12 Q. Zhang, B. Li, S. Huang, H. Nomura, H. Tanaka and C. Adachi, Efficient blue organic light-emitting diodes employing thermally activated delayed fluorescence, Nat. Photonics, 2014, 8, 326-332.

13 T. Hatakeyama, K. Shiren, K. Nakajima, S. Nomura, S. Nakatsuka, K. Kinoshita, J. Ni, Y. Ono and T. Ikuta, Ultrapure Blue Thermally Activated Delayed Fluorescence Molecules: Efficient HOMO-LUMO Separation by the Multiple Resonance Effect, Adv. Mater, 2016, 28, 2777-2781.

14 H. Ohkuma, T. Nakagawa, K. Shizu, T. Yasuda and C. Adachi, Thermally Activated Delayed Fluorescence from a Spiro-diazafluorene Derivative, Chem. Lett., 2014, 43, 306-308.

15 G. H. Kim, R. Lampande, J. B. Im, J. M. Lee, J. Y. Lee and J. H. Kwon, Controlling the exciton lifetime of blue thermally activated delayed fluorescence emitters using a heteroatom-containing pyridoindole donor moiety, Mater. Horiz., 2017, 4, 619-624.

16 J. Li, D. Ding, Y. Tao, Y. Wei, R. Chen, L. Xie, W. Huang and H. Xu, A Significantly Twisted Spirocyclic Phosphine Oxide as a Universal Host for High-Efficiency Full Color Thermally Activated Delayed Fluorescence Diodes, Adv. Mater, 2016, 23, 3122-3130.

17 T. Nishimoto, T. Yasuda, S. Lee, R. Kondoa and C. Adachi, A six-carbazole-decorated cyclophosphazene as a host with high triplet energy to realize efficient delayed-fluorescence OLEDs, Mater. Horiz, 2014, 1, 264-269.

18 Y. Zhao, C. Wu, P. Qiu, X. Li, Q. Wang, J. Chen and D. Ma, New benzimidazole-based bipolar hosts: highly efficient phosphorescent and thermally activated delayed fluorescent organic light-emitting diodes employing the same device structure, ACS Appl. Mater. Interfaces, 2016, 8, 2635-2643.

19 D. Zhang, M. Cai, Z. Bin, Y. Zhang, D. Zhang and L. Duan, Highly efficient blue thermally activated delayed fluorescent OLEDs with record-low driving voltage utilizing high triplet energy hosts with small singlet-triplet splittings, Chem. Sci., 2016, 7, 3355-3363.

20 L. S. Cui, J. U. Kim, H. Nomura, H. Nakanotani and C. Adachi, Benzimidazobenzothiazole-based bipolar hosts to harvest nearly all of the excitons from blue delayed fluorescence and phosphorescent organic light-emitting diodes, Angew. Chem., 2016, 55, 6864-6868.

21 T. Motoyama, H. Sasabe, Y. Seino, J. Takamatsu and J. Kido, An $\alpha$-Carboline-containing Host Material for High-efficiency Blue and Green Phosphorescent OLEDs, Chem. Lett., 2011, 40, 306-308.

22 H. Fukagawa, N. Yokoyama, S. Irisa and S. Tokito, Pyridoindole Derivative as Electron Transporting Host Material for Efficient Deep-blue Phosphorescent Organic Light-emitting Diodes, Adv. Mater., 2010, 22, 4775-4778.

23 Y. Im and J. Y. Lee, Effect of the position of nitrogen in pyridoindole on photophysical properties and device performances of $\alpha$-, $\beta$-, $\gamma$ - carboline based high triplet energy host materials for deep blue devices, Chem. Commun., 2013, 49, 5948-5950.

24 M. H. Tsai, Y. H. Hong, C. H. Chang, H. C. Su, C. C. Wu, A. Matoliukstyte, J. Simokaitiene, S. Grigalevicius, J. V. Grazulevicius and C. P. Hsu, 3-(9-Carbazolyl) carbazoles and 3,6-Di(9-carbazolyl)carbazoles as Effective Host Materials for Efficient Blue Organic Electrophosphorescence, Adv. Mater., 2007, 19, 862-866.

25 S. Ye, Y. Liu, J. Chen, K. Lu, W. Wu, C. Du, Y. Liu, T. Wu, Z. Shuai and G. Yu, Solution-Processed Solid Solution of a Novel Carbazole Derivative for High-Performance Blue Phosphorescent Organic Light-Emitting Diodes, Adv. Mater., 2010, 22, 4167-4171.

26 K. Masui, H. Nakanotani and C. Adachi, Analysis of exciton annihilation in high-efficiency sky-blue organic lightemitting diodes with thermally activated delayed fluorescence, Org. Electron., 2013, 14, 2721-2726.

27 A. R. Lee, J. Lee, J. Lee and W. S. Han, Silicon-based carbazole and oxadiazole hybrid as a bipolar host material for phosphorescent organic light-emitting diodes, Org. Electron., 2016, 38, 222-229. 